\title{
New trial of negative pressure wound therapy for obese parturients after caesarean raises more questions
}

Justin Brandt ${ }^{1}$ and Cande Ananth (STATS CONSULTS ONLY) ${ }^{1}$

${ }^{1}$ Rutgers Robert Wood Johnson Medical School

April 15, 2021

Mini Commentary - BJOG

New trial of negative pressure wound therapy for obese parturients

after caesarean raises more questions

Justin S. Brandt ${ }^{1}$ Cande V. Ananth ${ }^{2-5}$

Author affiliations

1. Division of Maternal-Fetal Medicine, Department of Obstetrics, Gynecology, and Reproductive Sciences, Rutgers Robert Wood Johnson Medical School, New Brunswick, NJ

2. Division of Epidemiology and Biostatistics, Department of Obstetrics, Gynecology, and Reproductive Sciences, Rutgers Robert Wood Johnson Medical School, New Brunswick, NJ

3. Cardiovascular Institute of New Jersey, Rutgers Robert Wood Johnson Medical School, New Brunswick, NJ

4. Environmental and Occupational Health Sciences Institute, Rutgers Robert Wood Johnson Medical School, Piscataway, NJ

5. Department of Biostatistics and Epidemiology, Rutgers School of Public Health, Piscataway, NJ

\section{Corresponding author}

Cande V. Ananth

Division of Epidemiology and Biostatistics

Department of Obstetrics, Gynecology, and Reproductive Sciences

Rutgers Robert Wood Johnson Medical School

125 Paterson Street, New Brunswick NJ 08901

Email:ananthcv@rwjms.rutgers.edu

\section{Conflicts of interest statement}

All authors declare: no support from any organisation for the submitted work; no financial relationships with any organisations that might have an interest in the submitted work in the previous three years; and no other relationships or activities that could appear to have influenced the submitted work.

\section{Funding statement}

No financial support was necessary for the preparation of this manuscript or acquiring data.

\section{ORCID}


Justin S. Brandt 0000-0002-3194-1087

Cande V. Ananth 0000-0002-0410-2595

Word count : 460

The obese parturient is at increased risk for numerous postoperative complications, including surgical site infections (SSI). Several promising strategies have emerged to reduce the risk of wound complications, including appropriately dosed antibiotics, antiseptic vaginal preparations, and prophylactic incisional negative pressure wound therapy (iNPWT). The latter approach is an innovation that applies negative pressure to a closed caesarean incision, redistributes lateral tension, reduces edema, and protects the surgical site from external infectious sources.

In this edition of the BJOG, Hyldig and colleagues (Prophylactic negative pressure wound therapy in obese women undergoing caesarean section: a commentary on new evidence that fuels the debate. BJOG $2021 \mathrm{xxx}$ ) have written a commentary about a recently published multicenter randomized trial of obese women in the United States who underwent caesarean delivery that compared iNPWT to standard wound dressings. The trial, which included 1624 patients recruited from February 2017 through November 2019, was prematurely stopped due to futility and concerns about serious adverse events (SAE). The authors concluded that iNPWT does not reduce the risk of SSI in obese women.

Hyldig and colleagues raise several methodological problems with the trial. A primary concern was related to the conclusion of "no difference" when the trial was powered as a superiority trial rather than an equivalence trial. The rate of SSI was less than expected, and Hyldig and colleagues argue this may have been a consequence of ineffective study design. Patients were consented for study participation when they were in labour, rather than before labour, which likely precluded participation of the obese patients at highest risk for SSI who could not be enrolled if/when an urgent indication for delivery arose, such as cord prolapse or category 3 fetal heart rate tracing due to abruption. The trial also reported higher than expected rates of SAE, including skin blisters, but many of these events have been characterised as mild events in other studies and may have been prevented with proper administration of the negative pressure device. They were concerned about another potential limitation of study design that may have biased the study's findings to the null. The iNPWT was left in place during hospitalisation for a median of 4 days while the iNPWT devices are designed for 7-days of continuous use.

Although Hyldig and colleagues have published extensively on the potential benefits of iNPWT and may have a favorable bias towards this intervention, their arguments are statistically sound and merit attention. The published trial provides level 1 evidence, but the study's conclusions raise more questions. Indeed, the results of the study should not dissuade the use of this intervention and, rather, should motivate the performance of a properly designed and powered superiority trial investigating the potential benefits of iNPWT to reduce the risk of surgical site infections for obese women. 